\title{
An optimization of protocol for mixed chimerism induction in mice model
}

\author{
M. Baśkiewicz-Masiuk1, K. Grymuła1, E. Pius'1, M. Hałasa1, V. Dziedziejko², \\ Ch. Schmidt' ${ }^{1}$ M. Walczak ${ }^{3}$, B. Machaliński1
}

${ }^{1}$ Department of General Pathology, Pomeranian Medical University, Szczecin, Poland

${ }^{2}$ Department of Biochemistry and Medical Chemistry, Pomeranian Medical University, Szczecin, Poland

${ }^{3}$ Clinic of Pediatrics, Pomeranian Medical University, Szczecin, Poland

\begin{abstract}
Studies on mixed chimerism are currently focused primarily on obtaining less toxic conditioning protocols. With these issues in mind, we have undertaken the attempt to optimize the procedure of mixed chimerism induction in mice. In order to reduce toxicity, we used decreasing doses of total body irradiation (TBI) together with combination of blocking antibodies. We also tried to eliminate immunosuppression (cyclophosphamide - CP) treatment after bone marrow transplantation. B6.SJL-Ptprc ${ }^{a} P e p 3^{b}$ mice were injected with 20-30 $\times 10^{6}$ bone marrow cells from Balb C mice. Mice were treated with TBI ( $3-1.5-0$ Gy) on "-1" day of the experiment and blocking antibodies against CD40L ("0", and "4" days) and additionally anti-CD8 ("-2" day) and/or anti-NK1.1 ("-3" day). Mice in certain groups also received CP (175 mg/kg) on "2" day. Presence of mixed chimerism was assessed in peripheral blood cells by flow cytometry on the $1^{\text {st }}, 2^{\text {nd }}, 3^{\text {rd }}, 4^{\text {th }}, 6^{\text {th }}$ and $8^{\text {th }}$ weeks of the experiment by detecting of CD45.1 (characteristic for B6.SJL-Ptprc ${ }^{a}$ Pep $3^{b}$ strain) and CD45.2 (characteristic for Balb $\mathrm{C}$ strain) antigens expression. We also analyzed the percentage of peripheral blood CD8 T-cells (CD3e/CD8a) and NK cells (Ly-49D/NK1.1). We found that reduction of TBI dose and elimination of CP decrease the rate of mixed chimerism formation. The highest percentage of donor cells was obtained in the group of animals treated with 3 Gy of TBI, CP and combination of anti-CD40L, anti-CD8, and anti-NK1.1 antibodies. The 3 Gy TBI was necessary to induce stable mixed chimerism, but it could be obtained without the CP use. The percentage of CD3e/CD8a and Ly49D/NK1.1 cells was significantly lower in the groups of mice treated by corresponding antibodies. Moreover, we observed the lowest number of peripheral blood Ly-49D/NK1.1 cells in the group of animals with highest mixed chimerism. Our experiments in mice model can help in better understanding of mixed chimerism phenomenon and in selecting the method of mixed chimerism induction with lowest possible toxicity. This also might improve the protocols of stable mixed chimerism induction in humans, and in the future, the effectiveness of vascularized organ transplantation.
\end{abstract}

Key words: CD3e/CD8a cells, Ly-49D/NK1.1 cells, mixed chimerism, TBI

\section{Introduction}

The phenomenon of mixed chimerism has been extensively studied for the last few years due to its potential effect on induction of alloantigens tolerance after transplantation of vascularized organs [1]. The tolerance towards graft can improve results of the surgery and allow avoiding or reducing of the long-lasting immunosuppression, hence the mixed chimerism raises such a

Correspondence: M. Baśkiewicz-Masiuk or B. Machaliński, Dept. of General Pathology, Pomeranian Medical University, Al. Powstańców Wlkp. 72, 70-111 Szczecin, Poland; tel.: (+4891) 4661546, fax.: (+4891) 4661548, e-mail: poziomka@med.pam.szczecin.pl; machalin@sci.pam.szczecin.pl great interest [1,2]. Induction of the phenomenon needs proper host preparation and haematopoietic cell transplantation. Unfortunately, conditioning of the patient for mixed chimerism can cause serious side effects related to destruction of haematopoietic and immunological system [2,3]. To avoid the complications of chimerism induction, many attempts have been made to establish the conditioning strategy which possess possibly lowest toxicity. Since, the toxicity substantially results from total body irradiation (TBI) and immunosuppressive therapy, researchers usually try to lower the dose of TBI and eliminate or decrease duration of immunosuppression. TBI dose could be reduced to 3 Gy by applying of specific anti-lymphocyte antibodies, namely anti-CD3, anti-CD4, anti-CD8, anti-NK, as well as co-stimulation blockade using anti-CD40L 
antibody with or without CTLA4Ig [1-5]. Previous experiments in animal model have shown the attempts of further reduction of TBI to 2 and 1 Gy [6]. Sometimes the TBI was even totally eliminated from the induction protocol, but to overcome this, a significantly higher number of transplanted haematopoietic cells had to be used to effectively induce the chimerism [7]. Although less toxic preconditioning is better tolerated by the treated patients, it can cause lower percentage of mixed chimerism, which results in instability and ineffectiveness of alloantigen tolerance induction $[2,6]$.

With all these issues in mind, we have undertaken the attempt to optimize the procedure of mixed chimerism induction in mice. Here we have analyzed the outcome of different preconditioning methods with application of: 1) various doses of TBI $(3-1.5-0$ Gy), 2) immunosuppresive drugs (CP $175 \mathrm{mg} / \mathrm{kg}-0$ ) after hematopoietic cell transplantation, 3) combination of co-stimulation blockade (anti-CD40L antibody) and/or antibodies against CD8a, and NK1.1 antigens to test their effectiveness in chimerism induction. We observed and assessed the kinetics of the mixed chimerism development in peripheral blood of the experimental mice for 8 week period.

Since many reports showed a significant role of CD8-lymphocytes and NK cells in graft rejection, we analyzed mice peripheral blood CD3e/CD8a and Ly49D/NK1.1 cells to confirm the efficiency of antibodies applied and to try to correlate the number of the cells with effectiveness of mixed chimerism induction.

\section{Materials and methods}

Mice. The experiments were performed on 12-16-week male Balb $\mathrm{C}\left(\mathrm{H}-2^{\mathrm{d}}, \mathrm{I}_{-} \mathrm{E}^{+}\right)$and B6.SJL-Ptprc ${ }^{a} P e p 3^{b}\left(\mathrm{H}-2^{\mathrm{s}}, \mathrm{I}^{-} \mathrm{E}\right)$ mice [8]. Mice were purchased from The Jackson Laboratory (Bar Harbor, ME, USA). The chosen mice strains have different isoforms of the CD45 antigen, CD45.1 (B6.SJL-Ptprc ${ }^{a}$ Pep $^{b}{ }^{b}$ ) and CD45.2 (Balb $\mathrm{C})$, which enables precise analysis of chimerism by flow cytometry [9]. Additionally, they possess different MHC class I and II antigens. Mice were kept under specific pathogen-free conditions in the BIO.S.A. Blower Unit Vent II (Ehret, Labor- and Pharmatechnik, Germany).

Inducing chimerism. Each group of experimental mice consisted of 3 animals. First, we induced chimerism in 3 groups (G1-G3) to establish optimal configuration of antibodies used to detect its effectiveness. Conditioning was based on total body irradiation of B6.SJLPtprc ${ }^{a} P e p 3^{b}$ mice with gamma chain $\left({ }^{60} \mathrm{Co}\right)$ in 3 Gray (Gy) dose on day $-1^{\text {st }}$ of the experiment. Cyclophosphamide (Endoxan, Baxter Oncology $\mathrm{GmbH}$, Germany) in dose of $175 \mathrm{mg} / \mathrm{kg}$ was given intraperitoneally (i.p.) on the day $+2^{\text {nd }}$. Additionally, animals were injected i.p. with blocking antibodies against mouse NK1.1 (AntiNK1.1 mAb, clone PK136, Becton Dickinson, USA), CD8a (AntiCD8a mAb, clone 53-6.7, Becton Dickinson, USA) and CD40L (Anti-CD154 mAb, clone MR1, Becton Dickinson, USA) as indicated in Table 1 for each group of animals. $30 \times 10^{6}$ of unseparated Balb $\mathrm{C}$ bone marrow cells were given intravenously on day 0 (in $0.2 \mathrm{~mL}$ PBS). The cells were obtained by flushing of femurs and tibias with cold PBS (Phosphate-buffered saline) with 2\% fetal bovine serum (FBS), L-glutamine and antibiotics. After isolation, erythrocytes were depleted by 15 -minute incubation with ammonium chloride containing lysing solution (BD Pharm Lyse, Lysing Buffer, BectonDickinson, USA) and were passed through a $40 \mu \mathrm{m}$ nylon mesh (Cell Strainer, BD Falcon, Becton Dickinson, USA). Cell washing was performed at $4^{\circ} \mathrm{C}$ each time. Because in the $1^{\text {st }}$ group of animals (antiNK1.1 and anti-CD40L $\times 2$ ) we could not obtain stable chimerism, we excluded the combination of antibodies used in this group from further studies. In the further part of the experiment, in the group G4 and G5 we applied identical combination of antibodies as in the groups G2 (anti-CD8a and anti-CD40L x 2) and G3 (anti-CD8a, antiNK1.1, and anti-CD40L) but we resigned of CP. In the groups G6-G9 we decreased doses of TBI to 1.5 Gy (G6 and G7) and 0 Gy (G8 and G9) (Table 1).

Detection of chimerism. A presence of mixed chimerism in the peripheral blood leucocytes was measured using flow cytometry in the $1^{\text {st }}, 2^{\text {nd }}, 3^{\text {rd }}, 4^{\text {th }}, 6^{\text {th }}$ and $8^{\text {th }}$ weeks after bone marrow cell infusion based on analysis of CD45.1 (B6.SJL-Ptprc ${ }^{a} P e p 3^{b}$ ) and CD45.2 (Balb C) antigens as previously described [8]. The data were collected with FACScan (Becton-Dickinson, USA) and analyzed using the CellQuest program. Shortly, mouse peripheral blood was collected to tubes with heparin from tail vain in the $1^{\text {st }}$, $2^{\text {nd }}, 3^{\text {rd }}, 4^{\text {th }}, 6^{\text {th }}$ weeks and from heart in the $8^{\text {th }}$ week. Erythrocytes were lysed with ammonium chloride solution for $15 \mathrm{~min}$. Remaining leucocytes were washed in PBS with $2 \%$ FBS by centrifugation (1200 rpm, $4^{\circ} \mathrm{C}, 5 \mathrm{~min}$ ) and then stained with fluorescein isothiocyanate (FITC)-conjugated anti-CD45.2 antibody (clone 104) and phycoerythrin (PE)-conjugated anti-CD45.1 antibody (clone A20). The cells were incubated with the antibodies for $30 \mathrm{~min}$ at $4{ }^{\circ} \mathrm{C}$, washed and fixed with $1 \%$ formalin $[9,10]$. Appropriate isotype antibodies were used as negative controls. Chimerism was analyzed in $2 \times 10^{4}$ cells by flow cytometry within total leucocytes gated based on FSC/SCC diagram (FACScan, BD, USA).

Analysis of CD3e/CD8a and Ly-49D/NK1.1 cells. In the $8^{\text {th }}$ week of the experiment peripheral blood cells were stained with fluorescein isothiocyanate (FITC)-conjugated anti-CD3e antibody (clone 145$2 \mathrm{C} 11$ ) and phycoerythrin (PE)-conjugated anti-CD8a antibody (clone 53-6.7) to assess CD3e/CD8a cells, and FITC-conjugated anti-Ly49D (clone 4E5) and PE-conjugated anti-NK1.1 (clone PK136) to analyze Ly-49D/NK1.1 cells (BD Pharmingen, Becton Dickinson, USA) as described above. Routinely, the $2 \times 10^{4}$ cells were analyzed by flow cytometry in lymphocyte gate (FACScan, BD, USA).

Ethical issues. All experiments were approved by the Local Ethical Committee and were performed in accordance with guidelines of laboratory animal care.

Statistical analysis. The arithmetical means and standard deviations were calculated using MS Excel v. 97. Next, non-parametric tests were used because of abnormal distributions of analyzed data (the Shapiro-Wilk test). Significances of changes in the chimerism kinetics were evaluated with the Friedman ANOVA and the Wilcoxon signed-rank tests. The Kruskal-Wallis ANOVA and the Mann-Whitney tests were used to compare the percentage of CD3e/CD8a and Ly-49D/NK1.1 cells between different groups of animals. Statistical significance was defined as $\mathrm{p}<0.05$.

\section{Results}

\section{Kinetics of chimerism in mice peripheral blood in effect of various conditioning protocols use}

We analyzed the percentage of mixed chimerism in the peripheral blood leucocytes in the $1^{\text {st }}, 2^{\text {nd }}, 3^{\text {rd }}, 4^{\text {th }}, 6^{\text {th }}$ and $8^{\text {th }}$ weeks of the experiment based on expression of 
Table. 1. The conditioning strategies.

\begin{tabular}{|c|c|c|c|}
\hline Group & $\begin{array}{l}\text { Dose of radiation } \\
\text { (TBI) }\end{array}$ & Dose of cyclophosphamide & Dose of antibodies \\
\hline G1 & $3 \mathrm{~Gy}$ & $175 \mathrm{mg} / \mathrm{kg}$ & $\begin{array}{l}\text { Anti-NK1.1 } 0.5 \mathrm{mg} \text { i.p. ("- } 3 \text { " day) } \\
\text { Anti-CD40L } 2 \times 0.5 \mathrm{mg} \text { i.p. ("0" and " } 4 \text { " days) }\end{array}$ \\
\hline $\mathrm{G} 2$ & $3 \mathrm{~Gy}$ & $175 \mathrm{mg} / \mathrm{kg}$ & $\begin{array}{l}\text { Anti-CD8a } 0.5 \text { mg i.p. ("-2" day) } \\
\text { Anti-CD40L } 2 \times 0.5 \text { mg i.p. ("0" and "4" days) }\end{array}$ \\
\hline G3 & 3 Gy & $175 \mathrm{mg} / \mathrm{kg}$ & $\begin{array}{l}\text { Anti-NK1.1 } 0.5 \text { mg i.p. ("-3" day) } \\
\text { Anti-CD8a 0.5 mg i.p. (“-2" day) } \\
\text { Anti-CD40L } 0.5 \text { mg i.p. ("0" day) }\end{array}$ \\
\hline G4 & 3 Gy & $(-)$ & $\begin{array}{l}\text { Anti-CD8a } 0.5 \text { mg i.p. ("-2" day) } \\
\text { Anti-CD40L } 2 \times 0.5 \text { mg i.p. ("0" and " } 4 \text { " days) }\end{array}$ \\
\hline G5 & $3 \mathrm{~Gy}$ & $(-)$ & $\begin{array}{l}\text { Anti-NK } 1.10 .5 \text { mg i.p. ("-3" day) } \\
\text { Anti-CD8a } 0.5 \text { mg i.p. ("-2" day) } \\
\text { Anti-CD40L } 0.5 \text { mg i.p. ("0" day) }\end{array}$ \\
\hline G6 & 1.5 Gy & $(-)$ & $\begin{array}{l}\text { Anti-CD8a } 0.5 \text { mg i.p. ("-2" day) } \\
\text { Anti-CD40L } 2 \times 0.5 \text { mg i.p. ("0" and " } 4 \text { " days) }\end{array}$ \\
\hline G7 & $1.5 \mathrm{~Gy}$ & $(-)$ & $\begin{array}{l}\text { Anti-NK } 1.10 .5 \text { mg i.p. (“-3" day) } \\
\text { Anti-CD8a } 0.5 \text { mg i.p. (“-2" day) } \\
\text { Anti-CD40L } 0.5 \text { mg i.p. (“0" day) }\end{array}$ \\
\hline G8 & $(-)$ & $(-)$ & $\begin{array}{l}\text { Anti-CD8a } 0.5 \mathrm{mg} \text { i.p. ("-2" day) } \\
\text { Anti-CD40L } 2 \times 0.5 \mathrm{mg} \text { i.p. ("0" and " } 4 \text { " days) }\end{array}$ \\
\hline G9 & $(-)$ & $(-)$ & $\begin{array}{l}\text { Anti-NK1.1 } 0.5 \text { mg i.p. ("-3" day) } \\
\text { Anti-CD8a } 0.5 \text { mg i.p. ("-2" day) } \\
\text { Anti-CD40L } 0.5 \text { mg i.p. ("0" day) }\end{array}$ \\
\hline
\end{tabular}

two isoforms of the CD45 antigen characteristic for each of the used mice strains (Fig. 1A). We obtained mixed chimerism in each mice of groups G1-G7, but in the last two groups of mice (G8-G9) we were able to induce only microchimerism (percentage of donor cells $<1 \%$ ) (Fig. 2). Among first three groups we observed the lowest percentage of donor cells in group G1 seen only in the $1^{\text {st }}$ week of the experiment, therefore we resigned of using combination of just anti-NK1.1 and anti-CD40L in further experiment. Comparing G2 and G3 groups we found the significantly higher chimerism in the group $\mathrm{G} 3$ of animals (3 Gy, CP, anti-NK1.1, antiCD8, anti-CD40L). The percentage of mixed chimerism was stable in the G2 and G3 groups during the entire time of experiment.

In next steps we resigned of $\mathrm{CP}$ and reduced TBI dose. As we expected, the degree of mixed chimerism correlated with applied immunosuppression and the dose of TBI. Elimination of CP lowered percentage of donor cells when comparing groups G3 and G5, but the differences were not statistically significant between groups G2 and G4. The great impact on chimerism induction and maintaining had the TBI dose. Decreasing it to 1.5 Gy lowered significantly percentage of chimerism as measured in each scheduled time interval. Resignation of TBI failed to induce mixed chimerism in the G8 and G9 mice.

\section{Analysis of mice peripheral blood CD8 T-cells (CD3e/CD8a) and NK cells (Ly-49D/NK1.1)}

We also investigated the percentage of $\mathrm{CD} 3 \mathrm{e} / \mathrm{CD} 8 \mathrm{a}$ and Ly-49D/NK1.1 cells in mice peripheral blood in the $8^{\text {th }}$ week of the experiment (Fig. 1B, 1C). We observed that each group of mice that received antiCD8a or anti-NK1.1 antibodies showed lower number of CD3e/CD8a $(\mathrm{p}<0.05)$ (Fig. 3) and Ly-49D/NK1.1 $(p<0.05)$ (Fig. 4) cells. Analysis of CD3e/CD8a cells demonstrated the lowest number of the cells in the G4 mice (3 Gy, anti-CD8, anti-CD40Lx2), however the difference was not statistically significant. The percentage of CD3e/CD8a cells did not correlate with the level and stability of mixed chimerism. However, we observed that the number of Ly-49D/NK1.1 cells was reduced in the G3 group where percentage of mixed chimerism was the highest.

\section{Discussion}

The main problem with obtaining mixed chimerism is toxicity of the pretreatment strategy. Pretreatment of host can cause many side effects like infection, risk of bone marrow relapse or graft versus host disease (GvHD). Until the problem is resolved, application of this method in tolerance induction will remain limited $[2,11]$. 


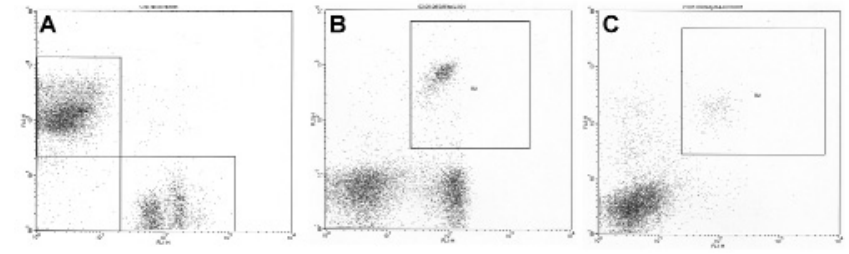

Fig. 1. Examples of flow cytometry analyses: A - chimerism (FL1 CD45.2-FITC - donor cells; FL2 - CD45.1-PE - recipient cells); B - the CD3e/CD8a cells (FL1 - CD3e-FITC; FL2 - CD8a-PE); C the NK1.1/Ly-49D cells (FL1 - Ly-49D-FITC; FL2 - NK1.1-PE)

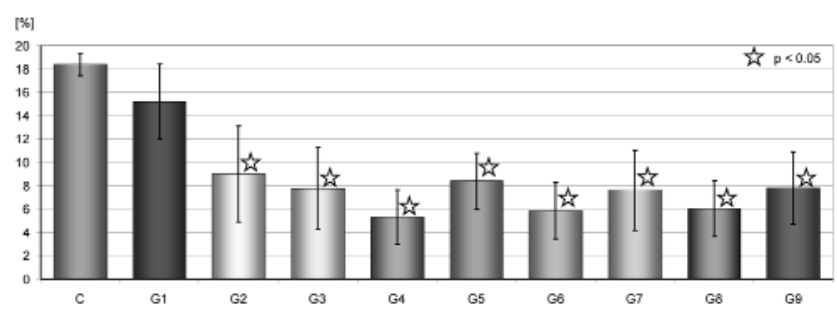

Fig. 3. The percentage of peripheral blood CD3e/CD8a cells in the $8^{\text {th }}$ week of the experiment.

First studies on mixed chimerism used myeloablative doses of TBI combined with immunosuppressive drugs $[2,12]$. Since that conditioning was toxic to the recipient, many affords have been made to lower the dose of TBI to 3 Gy. But decreasing the TBI dose needs, besides the use of immunosuppressive drugs, also the anti-lymphocyte antibodies treatment. Currently, large diversity of antibodies are applied in protocols of mixed chimerism induction, starting from anti-CD3, anti-CD4, anti-CD8 antibodies to commonly used costimulation blockade (anti-CD40L with/without CTLA4Ig) and previously reported anti-NK1.1 antibodies $[1-4,7,13]$. Besides the possibility of lowering the dose of TBI, antibodies can shorten the time of immunosuppression after transplantation. There are many of conditioning strategies that use 3 Gy of TBI, anti-lymphocyte antibodies and short-time immunosuppression. Recent reports suggest that TBI dose can be reduced to 2 or 1 Gy when combined with immunosuppressive drugs [6]. Also, the elimination of TBI was reported in the protocol which included only the costimulation blockade with antibodies, but that required injection of larger amount of haematopoietic cells $[2,7]$.

We started our experiment with the protocol based on $3 \mathrm{~Gy}$ of TBI and single injection of $175 \mathrm{mg} / \mathrm{kg}$ m.c. CP. We were applying standard doses of bone marrow cells, since higher doses are clinically unobtainable. We used anti-CD40L antibody, because many reports showed that this antibody inhibited effectively CD4 cells and helped inducing tolerance $[4,5,14]$. This antibody blocks second signal that is essential for full

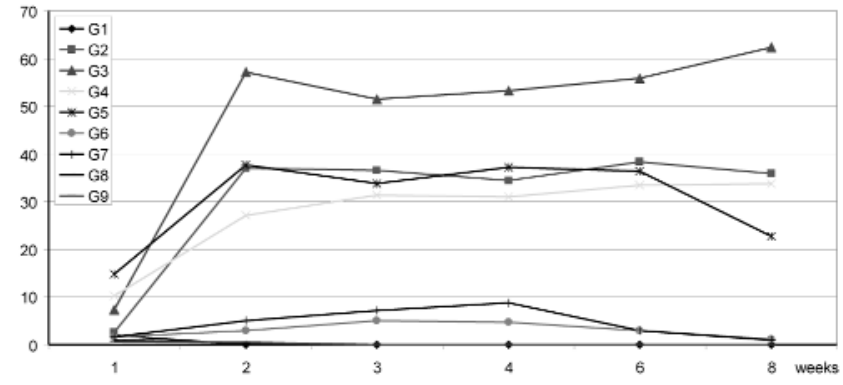

Fig. 2. Kinetics of chimerism in the peripheral blood leukocytes after various conditioning strategies applying.

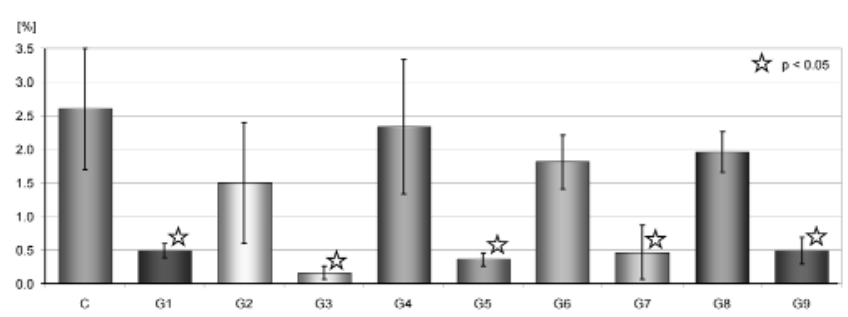

Fig. 4. The percentage of peripheral blood NK1.1/Ly-49D cells in the $8^{\text {th }}$ week of the experiment.

T-cell activation and differentiation of naive $\mathrm{T}$ cells into effector T cells $[1,2,5]$.

Besides CD4-lymphocytes, which play central role in alloresponse, the cytotoxic CD8-lymphocytes activated by $\mathrm{CD} 4$ cells also take part in graft rejection $[3,4,6]$. Since costimulation blockade did not prevent all of the CD8 cell-mediated allografts rejections, we used anti-CD8a antibody to block the CD8 cells $[5,13,15]$. Many researchers effectively applied this antibody in their induction protocols $[14,16]$. Anti-CD8 antibody with 3 Gy TBI and hematopoietic cell transplantation developed chimerism in CD40L $\mathrm{L}^{-/-}$mice [5]. On the other hand, elimination of the CD8 cells with anti-CD8 antibody did not improve hematopoietic cell engraftment in Westerhuis study [7]. The work of Takeuchi showed that moving the timing of 3 Gy TBI to day "-1" instead of day "0" of experiment allows for resignation of anti-CD8 antibody [15]. Our previous experiment suggested that in mice strains we used, which normally have high percentage of peripheral blood CD8 cells, the anti-CD8 antibody should be applied to obtain mixed chimerism [8]. The results obtained in the G1 group of animals, where combination of antibodies without anti-CD8 was not effective to induce stable chimerism, confirmed that the above suggestion is right.

Besides the $\mathrm{T}$ cells response to allograft, NK cells have been proven to participate in the rejection, especially of xenogeneic graft $[7,13]$. They are important in acute rejection, prior to $\mathrm{T}$-cell reaction against the hematopoietic stem cells [7]. The chimerism was also better induced after elimination of the NK cells 
[17-19]. Since, the anti-NK1.1 antibody was suggested to induce mixed chimerism without TBI [7], we also added anti-NK1.1 to our protocols to block the NK cells. In current experiment, highest percentage of donor cells was observed in mice that received combination of three antibodies in comparison to mice that were given anti-CD8a and two doses of CD40L (G3 vs. G2), which further suggests the influence of NK cells elimination on chimerism induction.

Another part of our experiment was the attempt to eliminate of immunosuppression treatment in conditioning protocols. Cyclophosphamid, applied in G1G3, induces immunoablation that helps to avoid graft relapse, but causes side effects, such like infection, mostly due to lymphopenia and lymphocyte suppression, but also neutropenia, cystitis, interstitial pneumonia, gonad and heart injury [20]. As we supposed, avoidance of immunosuppression decreased percentage of donor cells in chimeric mice. It was clearly observed in the G5 mice, where chimerism was significantly lower in comparison to G3, and decreased even more in the $8^{\text {th }}$ week of the experiment. In this group of animals anti-NK1.1 antibody was used instead of one dose of anti-CD40L. These results suggest that single dose of the CD40L antibody $(0.5 \mathrm{mg})$ without $\mathrm{CP}$ is not sufficient to block T-cell response. Consequently, this procedure is not as effective as in the G4 mice, where two doses of CD40L and anti-CD8 antibodies were applied. We state that induction of mixed chimerism is possible without immunosuppression but needs instead the set of precisely selected antibodies.

As a next step of the experiment we reduced the TBI dose to 1.5 Gy using previously established protocols with antibodies and without CP. Mixed chimerism was significantly lower in the G6 group (max. 5.05\%) as well as in the G7 mice (max. 8.76\%), and it diminished with the time of experiment. Moreover, elimination of TBI induced only the microchimerism in treated mice. We proved in present experiment, that reduction of the TBI dose did not give stable chimerism when immunosuppression was not used. Other authors' observations that showed possibility of TBI elimination may be explained by different mice strains combinations, since protocols using MHC-mismatched mice with the same genetic background provide higher potential of creating mixed chimerism. Another explanation may be larger amount of transplanted cells, even 10-fold of our dose, which for practical reasons was not possible to obtain [2]. To date, with some exceptions, TBI is essential in most of conditioning procedures. Although TBI is toxic to the patients, Sogawa et al. replaced it by short-term immunosuppression, and the 3 Gy TBI turned out to be more effective and less toxic than CP in $200 \mathrm{mg} / \mathrm{kg}$ m.c. dose [20].

In the $8^{\text {th }}$ week of our study we analyzed the CD3e/CD8a and the Ly-49D/NK1.1 cells levels in tested animals. We wanted to prove that antibodies we used in the experiment were effective in depleting of the CD8 T-lymphocytes and NK cells. We also wanted to test whether such elimination of the cells responsible for graft rejection plays any important role in mixed chimerism induction or maintenance. We expected that removing of CD8-lymphocytes was necessary to create mixed chimerism in our mice strain. However, we did not observe a correlation between number of the CD3e/CD8a cells and percentage of chimerism. Next, we analyzed the NK cells. Their elimination with the NK1.1 antibody improved chimerism induction in mice in which the CP treatment was used. On the other hand, in groups without $\mathrm{CP}$ use, this antibody probably did not effectively substitute the second dose of anti-CD40L, which effected in percentage of chimerism decrease in $8^{\text {th }}$ week of experiment. We applied the NK1.1 antibody, because Westerhuis showed that it could induce chimerism without TBI [7]. Unfortunately, this antibody blocks also the NK T cells, a subpopulation of T-lymphocytes that are thought to induce and maintain graft tolerance by their regulatory action, so the antibody can exert both of these somewhat contradictive effects $[17,21]$. The duality of the antibody influence in blocking the NK cells as well as the NK T cells could also explain our decreased chimerism in the $8^{\text {th }}$ week of experiment. Moreover, Shimizu et al. showed that use of the NK1.1 antibody induced higher level of chimerism but did not cause transplantation tolerance, which could also confirm our explanation [17].

Natural killer cells express activation receptor Ly49D, that mediates activation of NK-cell cytolysis. This receptor is not detected on NK1.1 T cells [17]. Previous study showed that NK cells expressed less Ly-49D antigen in tolerant mixed chimerism mice [19]. In our experiment, we observed that among animals that received the anti-NK1.1 antibody the lowest amount of the Ly-49D/NK1.1 cells was seen in the group with highest percentage of chimerism, so the Ly-49D/NK1.1 cells may become a good indicator of tolerance induced in chimeric mice.

To summarize, from among our different methods of chimerism induction, the most effective was one with 3 Gy TBI, CP and antibodies against CD40L, CD8a and NK1.1. The 3 Gy of TBI was necessary to obtain stable mixed chimerism, but it can be induced without immunosuppression (3 Gy TBI, 2x antiCD40L, antiCD8a). We proved the role of CD8 and NK cell elimination in mixed chimerism induction. Moreover, we suggest the decreasing of Ly49D/NK1.1 cells as the indicator of generated immune tolerance.

The experiments on mixed chimerism in mice model can help in better understanding of this phe- 
nomenon and in selection of best protocol for human purposes. The protocol can improve immune tolerance induction and therefore the effectiveness of vascularized organ transplantation. Moreover, it may help in avoiding of long-lasting immunosuppressive therapy.

Ackowledgements: Supported by a State Committee for Scientific Research as a grant N401 089 31/2056 to Magdalena Baśkiewicz-Masiuk from 2006 to 2009.

\section{References}

[ 1] Golshayan D, Buhler L, Lechler RI, Pascual M. From current immunosuppressive strategies to clinical tolerance of allografts: Transpl Int. 2007;20:12-24.

[2] Pree I, Pilat N, Wekerle T. Recent Progress in Tolerance Induction through Mixed Chimerism. Int Arch Allergy Immunol. 2007;144:254-266.

[3] Golshayan D, Pascual M. Tolerance-Inducing Immunosuppressive Strategies in Clinical Transplantation. Drugs. 2008; 68:2113-2130.

[4] Ito H, Takeuchi Y, Shaffer J, Sykes M. Anti-CD40L Monoclonal Antibodies Can Replace Anti-CD4 Monoclonal Antibodies for the Nonmyeloablative Induction of Mixed Xenogeneic Chimerism. Transplantation. 2006;82:251-257.

[ 5] Kurtz J, Ito H, Wekerle T, Shaffer J, Sykes M. Mechanism Involved in the Establishment of Tolerance Through Costimulatory Blockade and BMT: Lack of Requirement for CD40L-Mediated Signaling for Tolerance or Deletion of Donor-reactive CD4+ Cells. Am J Transplant. 2001;1:339-349.

[6] Luo B, Nanji SA, Schur CD et al. Robust Tolerance to Fully Allogeneic Islet Transplants Achieved by Chimerism with Minimal Conditioning. Transplantation. 2005;80:370-377.

[7] Westerhuis G, Maas WGE, Willemze R, Toes REM, Fibbe WE. Long-term mixed chimerism after immunological conditioning and MHC-mismatched stem cell transplantation is dependent on NK cell tolerance. Blood. 2005;106:2215-2220.

[ 8] Baśkiewicz-Masiuk M, Grymuła K, Hałasa M, Pius E, Boehlke $\mathrm{M}$, Machaliński B. Induction of mixed chimerism in mice by employing different conditioning protocols and bone marrow cell transplantation. Transplant Proc. 2009;41:1894-1899.

[ 9] Halasa M, Baskiewicz-Masiuk M, Dabkowska E, Machalinski B. An efficient two-step metod to purify very small embryonic-like (VSEL) stem cells from umbilical cord blond (UCB). Folia Histochem Cytobiol. 2008;46:239-244.

[10] Baumert B, Grymula K, Pietruszka D, et al. An optimization of hematopoietic stem and progenitor cell isolation for scientific and clinical purposes by the application of a new parameter determining the hematopoietic graft efficacy. Folia Histochem Cytobiol. 2008; 46:299-305.

[11] Fehr T, Sykes M. Clinical experience with mixed chimerism to induce transplantation tolerance. Transpl Int. 2008;21: 1118-1135.

[12] Colson YL, Lange J, Fowler K, Ildstad ST. Mechanism for Cotolerance in Nonlethally Conditioned Mixed Chimeras: Negative Selection of the V $\beta$ T-Cell Receptor Repertoire by Both Host and Donor Bone Marrow-Derived Cells. Blood. 1996;88:4601-4610.

[13] Murakami M, Ito H, Harada E, Enoki T, Sykes M, Hamano K. Long-Term Survival of Xenogeneic Heart Grafts Achieved by Costimulatory Blockade and Transient Mixed Chimerism. Transplantation. 2006;82:275-281.

[14] Kurtz J, Shaffer J, Lie A, Anosova N, Benichou G, Sykes M. Mechanisms of early peripheral CD4 T-cell tolerance induction by anti-CD154 monoclonal antibody and allogeneic bone marrow transplantation: evidence for anergy and deletion but not regulatory cells. Transplantation. 2004;103:4336-4342.

[15] Takeuchi Y, Ito H, Kurtz J, Wekerle T, Ho L, Sykes M. Earlier Low-Dose TBI or DST Overcomes CD8+ T-Cell-Mediated Alloresistance to Allogeneic Marrow in Recipients of AntiCD40L. Am J Transplant. 2004;4:31-40.

[16] Koyama I, Nadazdin O, Boskovic S et al. Depletion of CD8 memory $\mathrm{T}$ cells for induction of tolerance previously transplanted kidney allograft. Am J Transplant. 2007;7:1055-1061.

[17] Shimizu I, Tomita Y, Okano S et al. Efficacy and Limitation of Natural Killer Cell Depletion in CyclophosphamideInduced Tolerance. Surg Today. 2007;37:24-29.

[18] Cho SG, Shuto Y, Soda Y et al. Anti-NK cell treatment induces stable mixed chimerism in MHC-mismatched, T celldepleted, nonmyeloablative bone marrow transplantation. Exp Hematol. 2004;32:1246-1254.

[19] Kean LS, Hamby K, Koehn B et al. NK Cells Mediate Costimulation Blockade-Resistant Rejection of Allogeneic Stem Cells During Nonmyeloablative Transplantation. Am J Transplant. 2005;6:292-304.

[20] Sogawa H, Boskovic S, Nadazdin O et al. Limited Efficacy and Unacceptable Toxicity of Cyclophosphamide fort he Induction of Mixed Chimerism and Renal Allograft Tolerance in Cynomolgus Monkeys. Transplantation. 2008;86:615-619.

[21] Iwai T, Tomita Y, Shimizu I et al. The immunoregulatory roles of natural killer T cells in cyclophosphamide-induced tolerance. Transplantation. 2007;84:1686-1695.

Submitted: 6 June, 2009

Accepted after reviews: 5 August, 2009 